\title{
Dissipation and mass redistribution as a mechanism of propulsion in closed systems
}

\author{
Ademir L Xavier Jr*10 \\ ${ }^{1}$ Agência Espacial Brasileira, Brasília, DF, Brasil
}

Received on March 08, 2019; Revised on May 28, 2019; Accepted on June 04, 2019.

\begin{abstract}
In this work, the mechanism behind motion in closed systems is investigated by studying two simple mechanical systems. Firstly, a simple "ballistic" car system introduces the subject of propulsion as a function of a parameter that modulates the amount of ejected mass, thus allowing a transition between a closed to an open system. The second example provides a physically realizable (and optimizable) system, in which a mass sliding on the internal surface of a mechanical wheeled cavity is shown to produce a net movement, provided that dissipation is present. Several additional ways are discussed by which to 'open' a system whilst keeping the mass constant. As a function of strict momentum conservation, it is concluded that both internal mass motion and dissipation are key factors in the only way a mechanical system can move itself in a weak sense, in contrast to a strong meaning of 'self-propulsion'.
\end{abstract}

Keywords: closed systems, dissipation, propulsion, self-propulsion, reactionless drive.

\section{Introduction}

The subject of propulsion is traditionally introduced to students as a corollary of Newton's third law of motion and momentum conservation of a system with variable mass [1. Essentially, an 'open' system has part of its mass (consisting of chemical gases, ions and plasma) ejected to provide thrust to a main body carrying a useful load. The paradigm is the rocket, which has evolved throughout history of propulsion systems, whilst still keeping this same basic principle. New mechanisms have been proposed to circumvent the inherent inefficiency of this principle (as applied to rockets), in the context of interstellar trips [2,3] and to explore 'reactionless motion' (the idea of a self-propelled engine or the so-called 'space drive' 4,5$]$ ). As a concept, this kind of device embodies part of the hope for interstellar voyages because, using the rocket principle, it is impossible currently to carry all of the fuel necessary to reach the next star (and return). Some widely speculative ideas are based on violating mass conservation either by exploring the possibility of 'negative mass' [6], or by changing the system's overall inertial mass content 7 . A recent example of a potential 'breakthrough' was the discovery that electromagnetic waves in resonant cavities of a certain shape were observed to apparently generate thrust 8, 9, leading to criticism of the postulated reasons for the observed motion [10]. It is clear that the subject of propulsion and space travel provides a strong motivation for students to delve into the meaning of many fundamental laws of mechanics, such as momentum and energy conservation.

*Correspondence email address: ademir.junior@aeb.gov.br
The purpose of this paper is to present students a treatment of some simple mechanical systems that inspire the application of physics to the meaning of reactionless motion, i. e.: the possibility of motion without minor mass-ejection or system-splitting. One author $[2]$ stated recently:

"Because it would constitute a breakthrough to be able to move a vehicle without expelling a reaction mass, these devices appear to be breakthroughs",

which further motivates this investigation.

There have been many proposals in the past of mechanical oscillators which were claimed erroneously to produce thrust under non-reactive conditions $[11,12$. Such systems were promptly discredited, but misunderstandings concerning possible ways of motion under non-reactive conditions still remain. In the wake of the claims and criticism sparked by the EmDrive [8], other negative comments are found in popular media targeting the general public, e. g.: "Unless some kind of mass was being pushed out the back of this thing, an EmDrive based on any kind of propulsion simply shouldn't move itself though empty space" [13] and "Therefore, generating thrust from a closed system is impossible, which is why physicists have refused to accept the device from the start." [14].

We would like to elucidate the sense in which such statements should be understood, and emphasize the role played by momentum conservation in generating motion in a closed system when a certain amount of dissipation is present. In a certain sense or interpretation, a closed system can indeed 'propel itself'. However, this 
sense is not a strong one: no possible change in the system center of mass is possible. Dissipation and mass redistribution inside and within the system boundary play a fundamental role, together with momentum and energy conservation in generating a net displacement while keeping the overall mass of the system constant. The organization of this work is as follows: in Section 2 we introduced the ballistic car as a system which contains all the necessary ingredients to understand how motion of closed dissipative systems is possible. Section 3 and its two sections analyse in details a physical realization of the ballistic car. Generalizations are discussed in Section 4. The main conclusions are drawn in Section 5

\section{The ballistic car and the limit of a vanishing gunned wall}

A person firmly fixed on the flat car shots a bullet with mass $m$ at $t=0$ against a wall that is part of its platform (Figure 1). The combined mass of the person and flat car is $M$. From the standpoint of an observer fixed on the ground, the bullet speed is $\vec{v}=-|v| \hat{x}$. The wall intercepts a fraction of the bullet mass $m \zeta$ (with $0 \leq \zeta \leq 1$ ) at time $t=\tau_{c}$, while the remaining mass $m(1-\zeta)$ continues to move freely with velocity $\vec{v}_{e}=-\left|v_{e}\right| \hat{x}$ for $t \geq \tau_{c}$. Because the initial linear momentum of this system is zero, for $0 \leq t<\tau_{c}$ the car platform will move to the right with velocity magnitude

$$
V_{t<\tau_{c}}=\frac{\lambda}{1-\lambda}|v|
$$

with $\lambda=m /(m+M)$. The bullet trajectory for $0 \leq t<\tau_{c}$ is $x(t)=D-|v| t$, while the flat car moves according to $X(t)=V t$. The collision time is a solution of $x\left(\tau_{c}\right)=X\left(\tau_{c}\right)$ or $\tau_{c}=D(1-\lambda) / v$ with $D$ the gun-wall separation as shown in Figure 1. Hence, the total flat car displacement until $t=\tau_{c}$ is

$$
\Delta X=V_{t<\tau_{c}} \tau_{c}=\lambda D \text {. }
$$

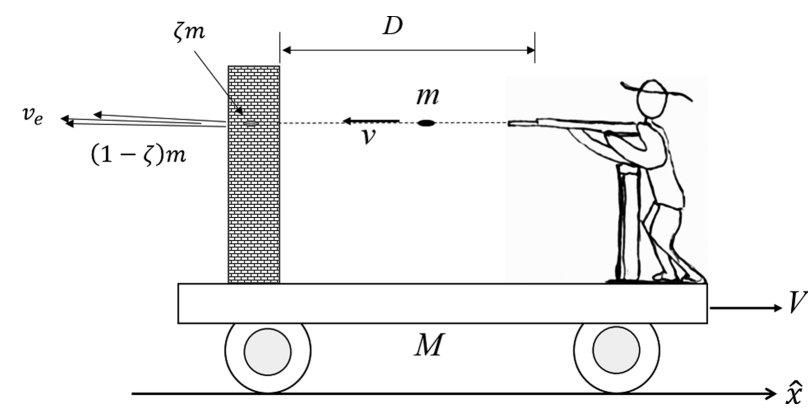

Figure 1: The 'ballistic car'. A person fixed on a flat car shots a bullet with mass $m$ and velocity $v$ against a wall that is part of the arrangement. The bullet hits its target at time $\tau_{c}$. After $\tau_{c}$, a fraction $m(1-\zeta)$ of the bullet continues to the left with velocity $v_{e}$ and the remaining $m \zeta$ is sunk in the wall.
The available internal energy of the system is provided by the shot explosion, being given effectively by

$$
E_{0} \leq \frac{1}{2}\left(\frac{\lambda}{1-\lambda}\right) M v^{2},
$$

as the energy fraction liberated by the explosion in the mechanical form. The conversion efficiency for $0 \leq t<\tau_{c}$ is the ratio of the kinetic energy of the main body with $V$ given by equation (1) and the initial energy given by equation (3)

$$
\epsilon_{t<\tau_{c}} \geq \frac{1 / 2 M V_{t<\tau_{c}}^{2}}{E_{0}}=\frac{\lambda}{1-\lambda} .
$$

For $t \geq \tau_{c}$, the final magnitude of the car's velocity follows again from momentum conservation taking into account the contribution of the remaining free mass (1ל) $m$ as

$$
V_{t \geq \tau_{c}}=\frac{(1-\zeta) \lambda}{1-(1-\zeta) \lambda}\left|v_{e}\right|
$$

The ejection velocity $v_{e}$ cannot be calculated by any simple relation, it is rather a function of the complicated interaction process of the bullet with the wall. Such relation can hardly be written down for a bullet penetrating a wall. In fact, the bullet-wall interaction (as well as the initial car acceleration with the recoil of the bullet shot) is assumed here to be instantaneous, hence the discontinuity in the derivative of the car velocity at $\tau_{c}$.

After $\tau_{c}$, the useful mechanical energy of the main body is simply $1 / 2(M+\zeta m) V_{t \geq \tau_{c}}^{2}$ or

$$
E_{m b}=\frac{1}{2} M v_{e}^{2} \frac{\lambda^{2}(1-\zeta)^{2}}{(1-\lambda)[1-\lambda(1-\zeta)]},
$$

from which we obtain the energy conversion efficiency $\epsilon=E_{m b} / E_{0}$ for $t \geq \tau$ as

$$
\epsilon_{t \geq \tau} \geq\left(\frac{v_{e}}{v}\right)^{2} \frac{\lambda(1-\zeta)^{2}}{[1-\lambda(1-\zeta)]} .
$$

Now we can compare two extreme cases:

- An open system, $\zeta=0$. The bullet velocity derivative is continuous at $\tau_{c}$ because the wall is completely transparent. Therefore equation (1) reduces to equation (5). It is also clear that $v_{e}=v$ and therefore $\epsilon_{t>\tau}=\epsilon_{t<\tau_{c}}=\lambda /(1-\lambda)$. The motion of the main body $\Delta X$ is unbounded and the dynamics correspond to the rocket system with a positive $\Delta v$, which is a velocity increment in case the flat car is observed from a moving reference system;

- A closed system, $\zeta=1$. The bullet collides completely with the wall and the final car velocity is zero (see equation (5)). The initial energy is completely absorbed by the wall so that $\epsilon_{t \geq \tau}=0$. The motion of the main body is restricted to the displacement $\Delta X$ until $\tau_{c}$. However, before this time 
the conversion efficiency is $\epsilon_{t<\tau_{c}}=\lambda /(1-\lambda)$, which may be interpreted as the proper efficiency of the system be it closed or not (that is, $\epsilon$ does not depend on $\zeta$ ). The effective motion of a closed system of this type is characterized by a positive ' $\Delta x$ ', but no $\Delta v$.

As $\zeta \rightarrow 0$, the wall vanishes and the system gradually opens. Both open and closed systems are extremes of boundary configurations that produce motion based on momentum conservation and mass redistribution. However, dissipation plays a relevant role only in closed systems because it is necessary to decelerate the redistributed particles in order to halt the motion with a net displacement while keeping the mass confined to the main body.

\subsection{Apparent thrust generated by the closed system}

In a rocket there is no absolute transfer of momentum, because no external force (except for gravity, when the rocket is launched from the surface of a massive body like Earth) acts on the whole system. The thrust $F$ is usually defined as the 'reactive force' [15] on the main body resulting from the ejection of particles of mass $m$ at rate $\dot{m}$ and velocity $v_{e}$

$$
F=\dot{m} v_{e}+A_{e}\left(P_{e}-P_{\infty}\right)
$$

with $A_{e}$ the nozzle exit area, $P_{e}$ the gas pressure at the nozzle exit and $P_{\infty}$ the ambient pressure. In propulsion engineering, the so-called 'specific impulse' [15] is a relevant parameter with which to characterize the performance of a thruster and is defined by

$$
I=\frac{\int_{0}^{t} F d t^{\prime}}{g \int_{0}^{t} \dot{m}(t) d t^{\prime}} .
$$

In a closed system containing mass redistribution and dissipation, both $\dot{m}$ and the total impulse are zero, and it it apparently meaningless to calculate the specific impulse for these systems, unless its definition is changed. The total impulse may be described as the result of an apparent or 'pseudo' thrust $\mathfrak{F}$ during the time $\tau_{c}$ with

$$
\mathfrak{F}=(M+m)\langle a\rangle \text {. }
$$

with $\langle a\rangle$ the average acceleration as given by

$$
\langle a\rangle=\Delta X / \tau_{c}^{2}
$$

in complete analogy with the thrust defined for a rocket. Using previous relations, for the ballistic car we find simply

$$
\mathfrak{F}=\frac{M \lambda v^{2}}{D(1-\lambda)^{3}}
$$

Finally, a new specific impulse $\mathfrak{I}$ for the closed systems should substitute the denominator in equation (7) as simply $m g$ or the weight of the moveable part so that

$$
\mathfrak{I}=\frac{\mathfrak{F} \tau_{\mathfrak{c}}}{m g} .
$$

For the closed ballistic car, the specific impulse is

$$
\mathfrak{I}=\frac{v}{g(1-\lambda)} .
$$

Another useful parameter is the thrust-to-power ratio [16] $\eta=\mathfrak{F} / \Pi$ with $\Pi$ the input power of the system. As the definition implies, $\eta$ is a measure how much thrust is generated in a propulsion system as a function of the input power. For the ballistic car, the input power may be approximated by $E_{0} / \tau_{c}$ (rate of the available initial energy to the interaction time) which, for the closed ballistic car is

$$
\Pi \approx \frac{\lambda M v^{3}}{2 D(1-\lambda)^{2}},
$$

and the thrust-to-power ratio is simply given by

$$
\eta \approx \frac{2}{v(1-\lambda)}
$$

For pedagogical reasons, it seems logical to define two ways by which a system of this type can move:

1. Propulsion in the strong sense: when the center of mass of the system moves, what can only happen when the system is truly open and actuated by an external force;

2. Propulsion in the weak sense: when no external force exists, the center of mass of the body remains stationary (as in the case of the rocket system). However, there is motion as measured, by instance, by the change in position of the 'geometric center' of the main body. Here, the word 'propulsion' is merely a synonym for the general motion observed with only part of the system, which is nevertheless a legitimate motion.

Thus, in spite of not being caused by an external agent, the motion of the ballistic car for $\zeta=1$ produces a tangible impression. If an external observer has no access to the car inner mechanism (implying a much stronger kind of closure), he would be surprised to detect a non zero displacement and would attempt to find perhaps an explanation based on an anomalous force.

\section{The dissipative mechanical cavity oscillator}

An example of a simple mechanical system implementing the ballistic car dynamics is depicted in Fig. 2. This figure shows a one dimensional mechanical oscillator 


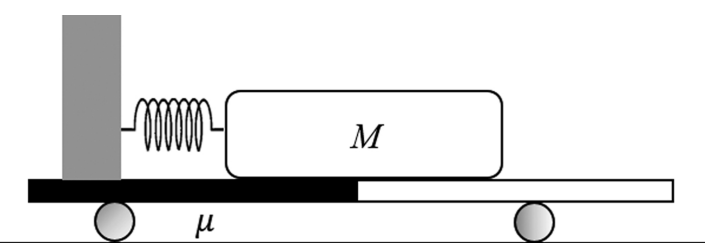

Figure 2: A possible equivalent implementation of the ballistic car: a mechanical oscillator of mass $M$ moves on a surface which is only half-dissipative (indicated by the friction coefficient $\mu$ ). The set is wheeled and and the observer registers the motion of the system geometric center.

on a surface which is only 'half dissipative'. The whole oscillatory cycle may be divided into several phases in which half is non dissipative and equivalent to shooting the bullet as in the previous example. The other half is dissipative and corresponds to the wall penetration by the bullet.

However, we explore here with some detail another mechanical version of the oscillator of Fig. 2, which has the advantage of being less artificial and easily replicated in the laboratory, providing an excellent way to study the effect of dissipation on the movement of closed systems. Engineering students will find it suitable for studies in system optimization because, as shown below, the maximum system displacement and apparent thrust is strongly linked to the kind of surface used to implement energy dissipation.

\subsection{Non dissipative motion of an oscillating cavity}

A free mechanical cavity of mass $M$ (Figure 3 is made of a cylindrical 'bow' of radius $R$ attached to a car that is free to move in the $x$-direction and a small body (a bead of mass $m$ ) constrained to move on its internal surface. In strict terminology, the system is open in the sense that gravity actuates along the $y$-axis but the cavity is restricted to move along $\hat{x}$ only. However, for all practical purposes, the system may be regarded as being 'closed along $x$ ' and of constant mass. The resulting motion can be emulated by a torsion pendulum with a mass $m$ and length $R$ carried by a car or wheeled platform in an environment for which $g=0$ (with $g$ being the gravity acceleration). The degree of freedom associated with the rotational motion of the bead is neglected, and the bead movement is restricted to the xy plane.

The car x-dynamics is referred to as a displacement $X(t)$ of the system center $O$ along $\hat{x}$. Besides the fixed xy system, a suitable polar system describes the bead position $\theta$ with the unitary vectors $(\hat{r}, \hat{\theta}), \hat{r}=\hat{x} \sin \theta-$ $\hat{y} \cos \theta$ and $\hat{\theta}=d \hat{r} / d \theta$. The Hamiltonian function of the

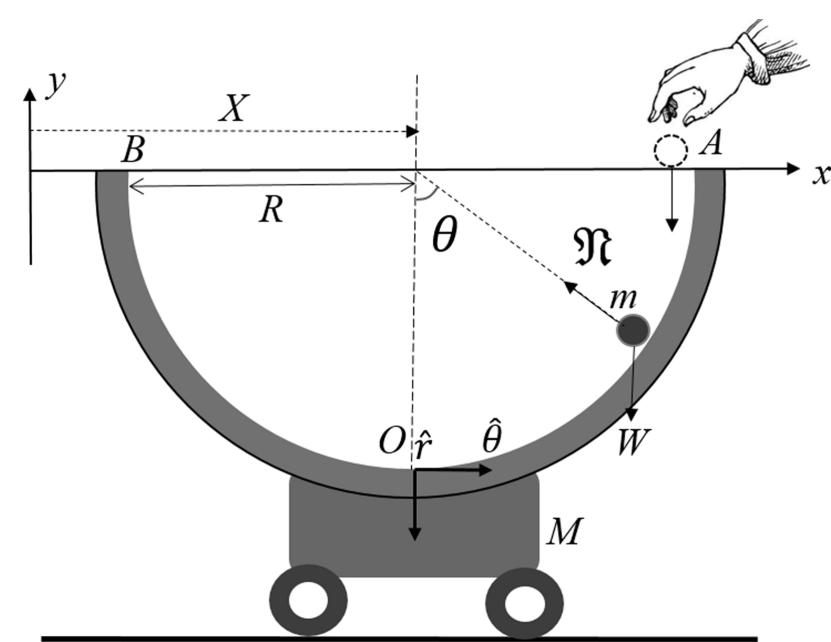

Figure 3: A mechanical cavity free to move along $\hat{x}$ under the inertial reaction of a slipping bead.

system under gravity acceleration $-g \hat{y}$ is

$$
\begin{aligned}
\mathfrak{H} & =\frac{h(\theta)(1-\lambda)}{2 M}\left(p_{X}^{2}+\frac{p_{\theta}^{2}}{\lambda R^{2}}-\frac{2 p_{\theta} p_{X} \cos \theta}{R}\right)- \\
& \frac{\lambda}{1-\lambda} M g R \cos \theta,
\end{aligned}
$$

with

$$
h(\theta)=\frac{1}{1-\lambda \cos ^{2} \theta},
$$

$p_{\theta}$ the angular momentum of the bead, $p_{X}$ the linear momentum of the cavity and $\lambda=m /(m+M)$ is the mass ratio. The vector $\left(X_{0}, P_{0}, \theta_{0}, \omega_{0}\right)$ represents the initial condition associated to the phase-space variables $(X, P, \theta, \omega)$, with $P=\dot{X}$ the cavity velocity along $\hat{x}$. A special initial state is $(0,0, \pi / 2,0)$ which corresponds to dropping the bead as shown in Figure 3 From equation (13), the canonical momenta of the system are explicitly given by

$$
\begin{aligned}
p_{X} & =\frac{M(P+\lambda R \omega \cos \theta)}{1-\lambda}, \\
p_{\theta} & =\frac{\lambda M R(R \omega+P \cos \theta)}{1-\lambda} .
\end{aligned}
$$

If the cavity is initially at rest, the numerical value of the first equation in 15$)$ is zero $(P=-\lambda R \omega \cos \theta)$ and remains so throughout the dynamic evolution. This is not true however for $p_{\theta}$, which is associated to an internal degree of freedom.

It is assumed that for all $\theta$ the bead is always in contact with the cavity surface, so that the normal force

$$
\mathfrak{N}=\frac{\lambda M}{1-\lambda}\left[R \dot{\theta}^{2}+(g+\dot{X} \dot{\theta}) \cos \theta\right]
$$

plays the role of centripetal force and is therefore directed toward $-\hat{r}$. The normal force function, equation (16), contains contributions of all accelerations on the bead: centripetal and gravity accelerations and $\dot{X} \dot{\theta} \cos \theta$ as 
an interaction term. Hence, the cases of interest are the ones for which $\mathfrak{N}>0$ restricting the three initial variables $\theta_{0}, \dot{\theta}_{0}$ and $\dot{X}_{0}$ (but not $X_{0}$ ). The system is equivalent dynamically to a pendulum attached to a moving pivot [17.

The equations of motion for the set of coordinates $(X, P, \theta, \omega)$ are

$$
\begin{aligned}
\dot{X} & =P, \\
\dot{P} & =\lambda h(\theta)\left(R \omega^{2} \sin \theta+g \sin \theta \cos \theta-\frac{f_{\theta}}{m R} \cos \theta\right), \\
\dot{\theta} & =\omega, \\
\dot{\omega} & =h(\theta)\left(\frac{f_{\theta}}{m R^{2}}-\lambda \omega^{2} \sin \theta \cos \theta-\frac{g}{R} \sin \theta\right) .
\end{aligned}
$$

Equations (17) are obtained from the $X$ and $\theta$ components of the forces under the evolution as defined by equations 13 plus an additional force $f_{\theta}$ along the $\hat{\theta}$ direction. These differential relations already consider the bead weight along $\hat{\theta}$ (the term $-g \sin \theta / R$ in the fourth equation in (17)), so that $f_{\theta}$ may represent other internal forces. For small amplitude angles $\left(\left|\theta_{0}\right| \lesssim 10^{\circ}\right)$, the bead moves as a simple pendulum with angular frequency $\Omega \approx \sqrt{g / R(1-\lambda)}$ that is higher than the equivalent frequency of an oscillation pendulum with length $R$. The oscillation period for the initial condition $(0,0, \pi / 2,0)$ is $T=c_{\lambda} \sqrt{2 R / g}$ with

$$
c_{\lambda}=\int_{-\pi / 2}^{\pi / 2} \sqrt{\frac{1-\lambda \cos ^{2} \theta}{\cos \theta}} d \theta
$$

from which the exact frequency $\Omega$ can be calculated.

For $\left|\theta_{0}\right| \leq \pi / 2$, the first equation of (17) is readily integrated to give the total displacement of the cavity center $\Delta X=X-X_{0}=\lambda R\left(\sin \theta_{0}-\sin \theta\right)$, which is equivalent to equation (2) in the ballistic car. Using the condition $(0,0, \pi / 2,0))$, the total displacement at $\theta=-\pi / 2$ is $2 \lambda R$ and zero upon returning to the initial position. The total average displacement as calculated by

$$
\begin{aligned}
\langle\Delta X\rangle & =\frac{1}{\theta-\theta_{0}} \int_{\theta_{0}}^{\theta} \Delta X\left(\theta^{\prime}, \theta_{0}\right) d \theta^{\prime} \\
& =\lambda R\left[\sin \theta_{0}+\frac{\left(\cos \theta-\cos \theta_{0}\right)}{\theta-\theta_{0}}\right],
\end{aligned}
$$

is half this value or $+\lambda R$ from $\theta_{0}=\pi / 2$ to $\theta=-\pi / 2$.

The available initial energy can be written as

$$
E_{0}=-\frac{\lambda}{1-\lambda} M g R \cos \theta_{0}
$$

which, for the specific initial condition $(0,0, \pi / 2,0)$ is zero and has a minimum for $\theta_{0}=0$. The kinetic energy of the system is easily written as

$$
E_{k}=\frac{1}{2} M R^{2} \omega^{2}\left[\frac{\lambda\left(1-\lambda \cos ^{2} \theta\right)}{1-\lambda}\right] .
$$

In the absence of friction, the entire system will oscillate on average $\lambda R$ with no net displacement (limit of periodic motion $\theta \rightarrow \theta_{0}$ in equation (19)), which is commonly interpreted as a consequence of no external force driving the main system of mass $M+m$. The frictionless wheeled cavity cannot therefore produce any net motion.

\subsection{Dissipative motion}

Friction is one of the forces that may be represented by $f_{\theta}$ in equations (17). Bearing in mind the distinction between static and dynamic dry friction [18, the following dissipative force model may be applied

$f_{\theta}=-\mu \operatorname{sgn}(\omega) \mathfrak{N}(P, \theta, \omega) \Theta\left(|\omega|-\omega_{m}\right) \Theta\left(\theta_{1}-\theta\right) \Theta\left(\theta-\theta_{2}\right)$,

with $\mu \geq 0, \Theta(x)$ the Heaviside function and $\operatorname{sgn}(x)$ the signal function. The force domain is delimited by angles $\theta_{1}$ and $\theta_{2}$. Also, $\omega_{m}$ is the minimum angular bead velocity above which dynamical friction takes place. $\theta_{1}$ and $\theta_{2}$ are the leftmost and rightmost limits of the friction domain, respectively, with $\left|\theta_{1}\right| \leq \pi / 2$ and $\left|\theta_{2}\right| \leq \pi / 2\left(\theta_{2}<\theta_{1}\right)$. The force direction is given by the signal of $\omega$ through $\operatorname{sgn}(x)$. For example, if $\theta_{1}=\pi / 2$ and $\theta_{2}=-\pi / 2$ the cavity is entirely dissipative.

The dissipative motion is illustrated by a straightforward numerical integration of equations (17) using equation 22 , and by assuming $\theta_{1}=0$ and $\theta_{2}=-\pi / 2$, so that the friction domain is along the segment $\overline{O B}$ in Figure 3. The value $\omega_{m}=0.001 \mathrm{rad} / \mathrm{s}$ is taken as the minimum angular velocity in equation 22. A typical time evolution of the system is shown in Figure 4 (top) for $\lambda=1 / 6(m=2 \mathrm{~kg}, M=10 \mathrm{~kg}$ and $R=1 \mathrm{~m})$ and $\mu=$ 0.2 . For these assumed numerical parameters, the cavity period is $T=2.301 \mathrm{~s}$. The cavity phase-space evolution $(X, P)$ is seen in the spiral of Figure 4 (bottom). Dashed lines correspond to undamped movement $(\mu=0)$. The letters in this figure are references to the sections of the bead trajectory in Figure 3 . Segment $\overline{A O}$ is the initial undamped motion. The dissipative force actuates along $\overline{O B}$. The return to sector $\overline{A O}$ occurs only if sufficient energy is available, as is the case with $\mu=0.2$.

Contrary to the conservative case, the dissipative cavity never returns to the initial condition $(0,0, \pi / 2,0)$, but relaxes to $\left(\Delta X_{d}, 0, \theta_{d}, 0\right)$ after a certain time $\tau_{d}$ (as indicated in Figure 4 (Top), $\tau_{d}=4.836 \mathrm{~s}$ ). The total displacement is $\Delta X_{d}=0.186 \mathrm{~m}$. From the point of view of energy balance, dissipation converts part of the available initial energy, Eq. 20), into motion during $\tau_{d}$ with an average velocity $\langle P\rangle \simeq \Delta X_{d} / \tau_{d}=0.038 \mathrm{~m} / \mathrm{s}$.

The 'useful' kinetic energy is $M\langle P\rangle^{2} / 2(1-\lambda)$, from which the conversion efficiency $\epsilon \approx \Delta X_{d}^{2} /\left(2 g \lambda R \tau_{d}^{2}\right)$ is determined as the rate between the generated energy and the initially available one as given by equation 20 . For the parameters of the simulated case $\epsilon_{\mu=0.2}=0.045 \%$. For weak friction $(\mu \ll 0.1)$, the minimum energy is close to $E_{0}$, equation 20 , that is $\theta_{d} \approx 0$ with a long relaxation 

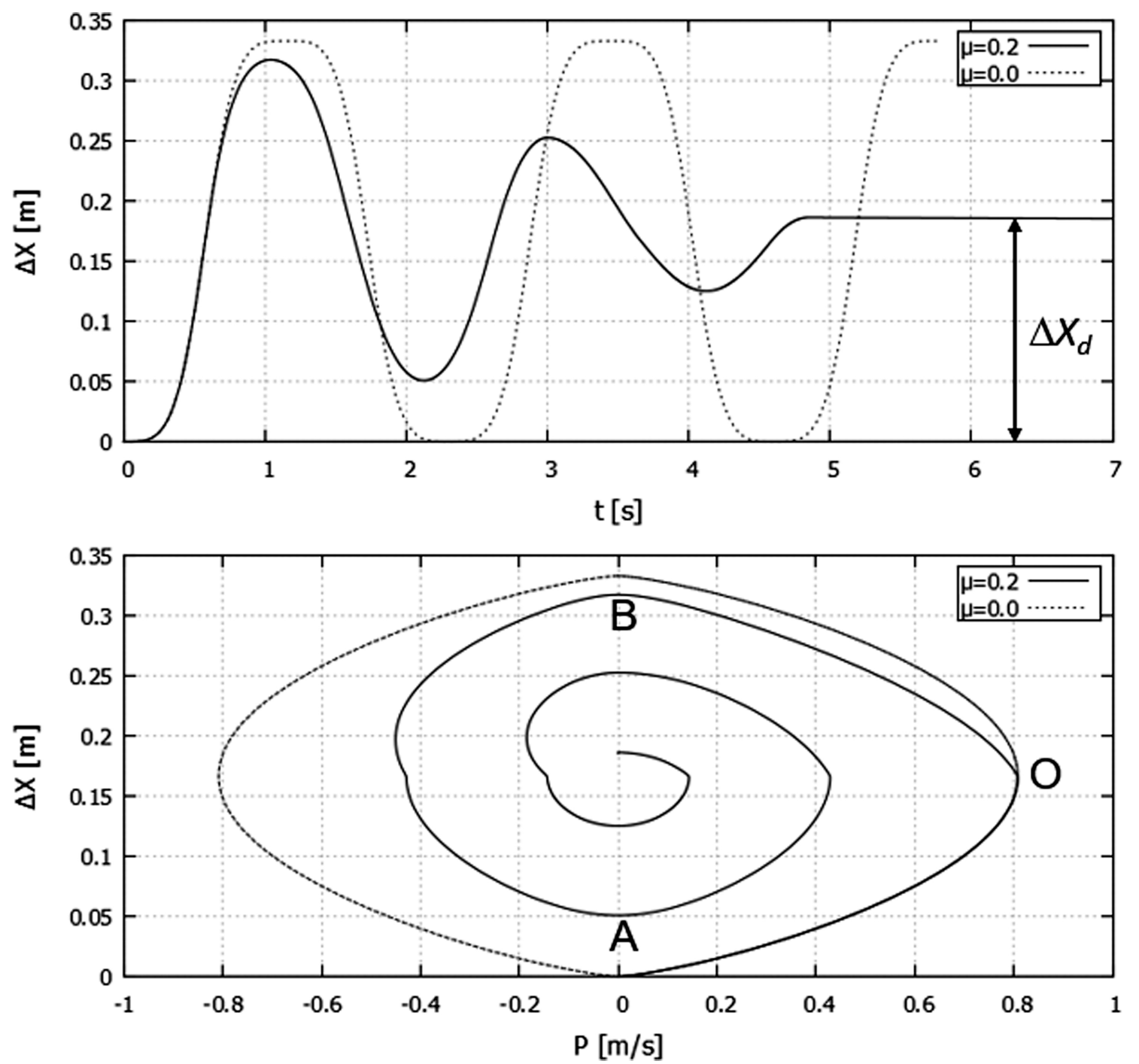

Figure 4: Numerical integration for the dissipative cavity for $\lambda=1 / 6, M=10 \mathrm{~kg}$ and $\mu=0.2$. Top: Cavity center evolution against the undamped case $(\mu=0$, dashed line). Bottom: $(X, P)$ plot showing the undamped and friction phases of motion. The indicated $\Delta X_{d}=0.186 \mathrm{~m}$ is the net cavity displacement which takes place during $\tau_{d}=4.836 \mathrm{~s}$

time and low $\epsilon$. For $\mu \gg 1$, the bead may be treated as colliding inelastically with the dissipative surface and again $\theta_{d} \approx 0$. However, the relaxation time is the shortest possible, close to $1 / 4$ of the non dissipative period and the efficiency $\epsilon \approx 4 \lambda / c_{\lambda}^{2}$, which is equivalent to equation (4) of the ballistic car. Between these two extremes, $\theta_{d}$ oscillates with eventual values of $\mu$ for which $\theta_{d}$ is exactly zero. Thus, the relaxed state does not correspond to the absolute energy minimum $E_{0}$.

The evolution of the acceleration is well appreciated in the cavity system, for which a specific dissipative interaction is given. Figure 5 shows the evolution of cavity velocity (left) and acceleration (right), which is proportional to the cavity apparent thrust. However, the average acceleration as calculated by $\int_{0}^{\tau} \dot{P} d t=0$ (the area below the curve in Figure 5 (Right)), because both the initial and final cavity velocities are zero. Thus the apparent thrust, as a performance parameter, should be calculated from the overall displacement and relaxation time as $(M+m) \Delta X / \tau_{c}^{2}$. In the case treated in the last $\operatorname{section}(\lambda=1 / 6$ and $\mu=0.2) \mathfrak{F}_{c}=0.095 \mathrm{~N}$ and the total impulse is $0.46 \mathrm{Ns}$.

Just as in the case of the ballistic car, for the cavity with $\mu \gg 1$, the apparent thrust is

$$
\mathfrak{F}_{c} \approx \frac{8 \lambda}{(1-\lambda) c_{\lambda}^{2}} M g
$$

the thrust-to-power ration is

$$
\eta_{c} \approx \frac{2}{c_{\lambda}} \sqrt{\frac{2}{R g}}
$$

and the specific impulse is

$$
\mathfrak{I}_{c} \approx \frac{2}{c_{\lambda}} \sqrt{\frac{2}{R g}} .
$$



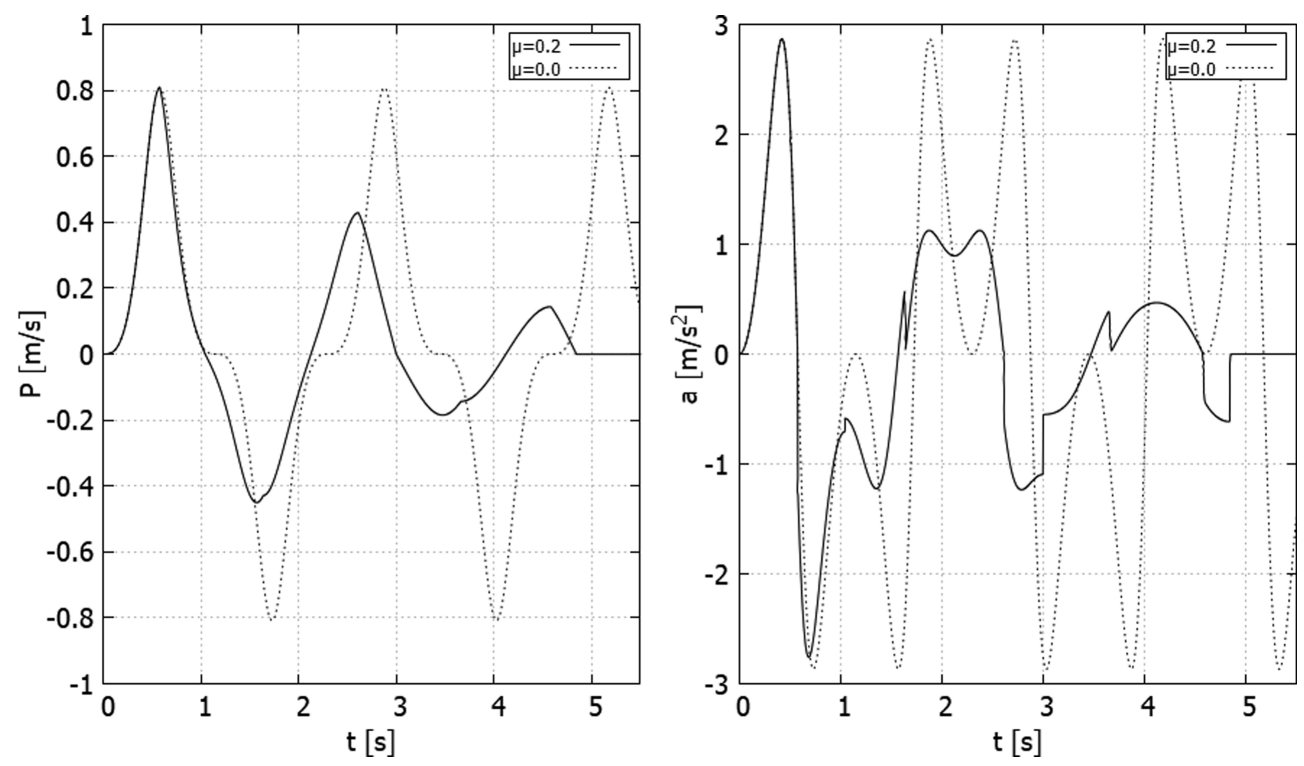

Figure 5: Time evolution of the cavity velocity (left) and acceleration (right) for $\mu=0.2$. The dotted curve corresponds to the undamped case. The average acceleration using $\langle a\rangle=\Delta X / \tau_{d}^{2}$ is $1 / 1233 \mathrm{~g}$ and $\langle P\rangle=0.038 \mathrm{~m} / \mathrm{s}$.

Dimensionally both equation (24) and 12 have unit $\left[v^{-1}\right]=\mathrm{s} / \mathrm{m}=\mathrm{N} / \mathrm{W}$. Using the output of the case calculated in the previous section (for which equations above are not valid), $\Pi_{c}=4.05 \mathrm{~W}, \mathfrak{I}_{c}=0.025 \mathrm{~s}$ and $\eta_{c}=$ $23.5 \mathrm{~N} / \mathrm{kW}$. For the optimized case, one obtains instead: $\Delta X_{\mu>>1}=0.167 \mathrm{~m}, \tau_{\mu>>1}=0.576 \mathrm{~s},\langle P\rangle_{\mu>>1}=0.289$ $\mathrm{m} / \mathrm{s},\langle a\rangle_{\mu>>1} \approx 1 / 19 \mathrm{~g}, \mathfrak{F}_{\mu>>1}=6.02 \mathrm{~N}$ (equation (23)), $\Pi_{\mu>>1}=34 \mathrm{~W}, E_{k \mu>>1}=0.5 \mathrm{~J}, \epsilon_{\mu>>1}=2.5 \%, \mathfrak{I}_{\mu>>1}$ $=0.177 \mathrm{~s}$ (equation (25) and $\eta_{\mu>>1}=176.9 \mathrm{~N} / \mathrm{kW}$ (equation (24)). Contrary to the undamped case, the dissipative cavity does does produce average motion, because its net displacement is not zero but $\Delta X_{d}$.

\section{Discussion}

In fact, the total displacement observed in the dissipative systems treated here can be decomposed into several small mass contributions as shown in Figure 6 (A), in which a 'mass tank' (a) is depleted continuously as shown for the cavity system. The displaced beads are kept in the cavity base (b). The equivalent process in the ballistic car is the shooting of several bullets toward the wall with $\zeta=1$. One can ask if it is possible to reuse the masses as shown in Figure 6 (B), in which an internal elevation mechanism (d) replenishes the tank and recharges the system energy. However, it is easily seen that such mass recycling does not lead to any net displacement. If there are $N$ beads and the unit displacement is $m R /\left(M_{0}+\right.$ $N m$ ), then the overall shift in position to the right is $N m R /\left(M_{0}+N m\right)$, with $M_{0}$ the empty cavity mass. Mass recycling would lead to a net shift to the left by the same amount and no overall motion. The only way to take advantage of the same masses is by fastening the whole vehicle to the ground during mass recycling as shown in (e), which corresponds to applying an external force to the system. The system would be open then, just as represented in Figure 6 $(\mathrm{C})$, where there is no mass storage or external force, but the beads are dropped by an external agent and scape through an exit (g) as in the rocket system, keeping the mass of the system constant. Note that, in this case, in spite of the similarity with the rocket, there is no momentum gain along $\hat{x}$ because the mass motion in the hole is along $\hat{y}$.

\section{Conclusions}

Propulsion is usually taught as a kind of energy conversion and mass loss process in which particles of certain mass and average velocity produce an acceleration of the main body, counterbalancing external forces such as gravity. Momentum is conserved rigorously for the whole system (body + particles), and the 'momentum transfer' is, in fact, a consequence of fixing the attention to the main body or regarding motion of only some of its parts. By the same principle, a closed system (with constant mass and no external force) may displace itself in space in a weak sense.

However, the price paid for motion in a closed system is quite high: it lasts no longer than a short interval $\tau_{c}$, roughly of the order $l / v$, with $l$ a system size and $v$ the internal velocity of the movable part. It is reasonable to speak about the system as possessing a 'displacement cycle' characterized by a net " $\Delta X$ " and $\tau_{c}$, during which all motion takes place. Worst still, the final system state has no $\Delta v$, implying in no net momentum change.

Although no net momentum change exists after the displacement cycle, it is possible to define an average transient equivalent impulse during motion as $\sim(M+$ $m)\langle a\rangle \tau_{c}$, which is a measure of the strength of the "pseudothrust' as defined by $(M+m)\langle a\rangle$. This designation em- 


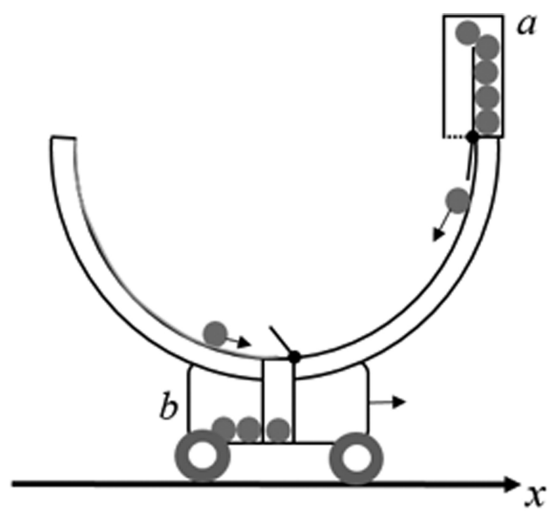

(A)

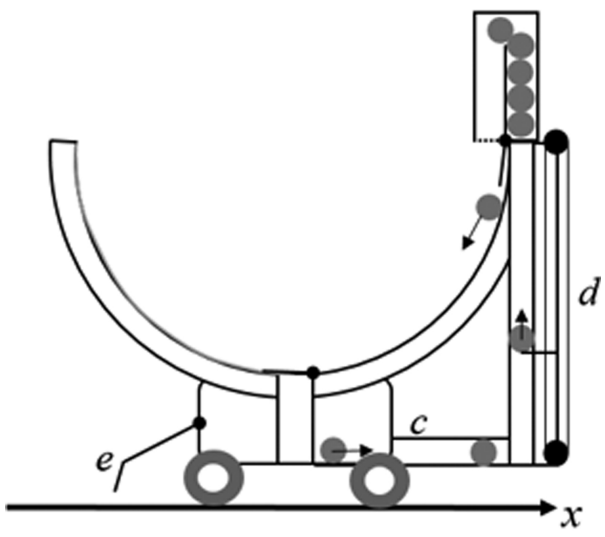

(B)

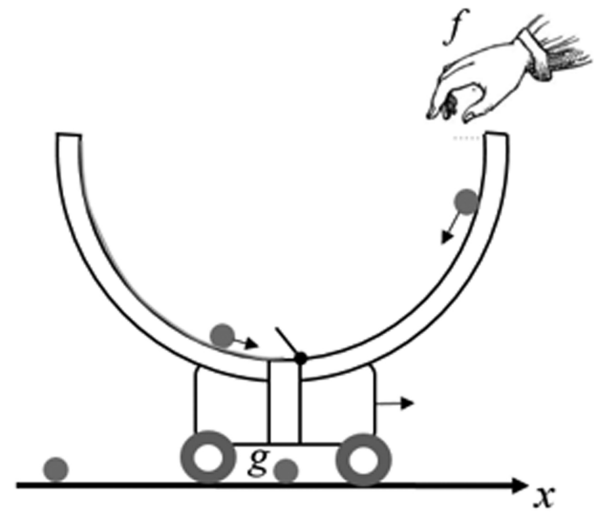

(C)

Figure 6: In (A) the system is closed but displacement is limited by the number of total beads in the 'mass tank' (a); in (B) an elevation and 'recharging' mechanism (d) would attempt to reuse the masses through an internal transport mechanism as shown in (c). However, in this case, there is only net motion (to the right) if the car is fastened to the ground (e). In (C), the masses are allowed to leave the cavity through a hole $(\mathrm{g})$ and an external agent $(f)$ recharges the system.

phasizes that the thrust does not originate from any action imposed by an external cause. However, an observer would see the closed system move as if impelled by a peculiar force, rendering the linear momentum unchanged. Because friction can drive out energy from the mechanical motion, the measurable result for the observer is a displacement of the entire body.

The functioning of such devices is strongly limited in its 'closure' definition: an attempt to reuse the same displaced masses in another displacement cycle is forbidden, because they would eliminate any effect from the previous cycle. Mass recycling is possible when either a force is applied in the opposite direction to cancel out the effect of the reusable masses' movement, or if a continuous flow of masses is allowed to enter and exit the system so as to keep its mass constant. For obvious reasons, a system implementing mass recycling in these ways would be anything but closed.

\section{Acknowledgments}

I gratefully acknowledge Dr. Jeff Pursglove for revising the article manuscript and an anonymous reviewer for a careful reviewing work.

\section{References}

[1] D. Halliday, R. Resnick and J. Walker, Fundamentals of Physics (John Wiley \& Sons, New Jersey, 1993).

[2] M.G. Millis and E.W. Davis, Frontiers of Propulsion Science (American Institute of Aeronautics and Astronautics, Virginia, 2009), p. 250.

[3] https://arxiv.org/abs/1101.1063.

[4] R. Sedwick and H. White, Journal of Spacecraft and Rockets 55, 1034 (2018).

[5] M.G. Millis, Journal of Propulsion and Power 13, 577 (1997).

[6] R.L. Forward, Journal of Propulsion and Power 6, 28 (1990).

[7] K.H. Wanser, Journal of Space Exploration 2, 121 (2013).

[8] H. White, P. March, J. Lawrence, J. Vera, A. Sylvester, A.D. Brady and P. Bailey, Journal of Propulsion and Power 33, 30 (2017).

[9] J. Mullins, New Scientist 191, 30 (2006).

[10] M. Tajmar, M. Kößling, M. Weikert and M. Monette, in Proc. of Space Propulsion Conference (Seville, Spain, 2018).

[11] N.L. Dean, US Patent 2,886,976 (1959).

[12] R.E. Foster, US Patent 5,685,196 (1997).

[13] https://www.sciencealert.com/impossible-em-drive-testconcludes-external-thrust.

[14] https://www.popularmechanics.com/science/ energy/a20896657/new-study-doubt-impossibleem-drive/

[15] G.P. Sutton and O. Biblarz, Rocket Propulsion Elements (John Wiley \& Sons, New Jersey, 2001).

[16] J. Lukas, G. Teel, J. Kolbeckand and M. Keidar, AIP Advances 6, 025311 (2016).

[17] M.R. Spiegel, Theory and Problems of Theoretical Mechanics (McGraw-Hill Brook Company, New York, 1967).

[18] B.N. Persson, Sliding friction: physical principles and applications (Springer Science \& Business Media, Berlin, 2013), 